\title{
Speckle observations of composite spectrum stars: II. Differential photometry of the binary components
}

\author{
J.-L. Prieur, J.-M. Carquillat, N. Ginestet, L. Koechlin, \\ UMR 5572, Observatoire Midi-Pyrénées - CNRS, 14 Avenue E. Belin, 31400 Toulouse, France. \\ and \\ A. Lannes, E. Anterrieu, \\ CERFACS, 42, Avenue Gaspard Coriolis, 31057 Toulouse Cédex, France. \\ and \\ S. Roques, \\ UMR 5572, Observatoire Midi-Pyrénées - CNRS, 14 Avenue E. Belin, 31400 Toulouse, France. \\ and \\ E. Aristidi, \\ UMR 6525, Université de Nice Sophia-Antipolis - CNRS, Parc Valrose, 06108 Nice Cedex 2, France. \\ and \\ M. Scardia \\ Osservatorio Astronomico di Brera, Via E. Bianchi 46, 22055 Merate, Italy.
}

\begin{abstract}
Multicolor differential photometry measurements of the two components of 18 close binary stars, mostly composite spectrum stars, are presented. They are based on observations made at Pic du Midi Observatory with the speckle camera PISCO between 1993 and 1998. Optical bench experiments were also performed to assess the validity of the whole process from the data acquisition with the ICCD detector to the final photometry measurements.

The results are discussed and compared with (i) Hipparcos and speckle photometry, (ii) spectroscopy measurements and (iii) composite spectra computed with a spectral library. Our measurements are in good agreement with other observations. We also determined the evolution stage of the individual components: the absolute visual magnitudes of the cool giant stars that we found are compatible with the calibrations made by other authors. This work shows that PISCO is well adapted to efficiently perform relative photometry of close binary stars.
\end{abstract}

Subject headings: Techniques: interferometric - Stars: binaries: close, visual - Stars: composite spectrum

\section{Introduction}

Composite spectrum stars are binary stars (often spectroscopic binary stars) generally composed by the association of a dwarf hot star (B or A-type) with a cool evolved star (G, K or M) (Ginestet et al. 1992). The study of these systems is of great astrophysical interest (i) to reach a better estimation of the masses of red giant stars and (ii) to constraint stellar evolution models. The main observational problem concerns the difficulty for identifying the spectral type of the two components separately.

When we started this project in the early 1990's, the spectral type of the components of most of these systems was erroneous. Indeed, in the conventional spectral domain of the MK classification $(380-400 \mathrm{~nm})$, the spectra of the two components appeared inextricably tangled and, as a consequence, the hot components were classified as too late and the cool components as too early. 
To establish the nature of the cool component we undertook a spectral survey in the near infra-red with the Carélec and Aurélie spectrographs at the Haute-Provence Observatory (Ginestet et al. 1994, 1997, 1999; Carquillat et al. 1997). The classification of the hot components in the near-UV by a "subtraction of spectra" method is presented in Ginestet et al. (2002).

In a previous paper (Prieur et al., 2002, Paper I), we presented our sample and the position measurements of the binary components. We report here our measurements of the differential photometry obtained with PISCO at Pic du Midi. As discussed by many authors (e.g., Roberts, 1999, and for a review: Worley et al., 2001), photometric measurements of individual stars in close binary systems are very difficult to perform: atmospheric turbulence, photon noise and detector defects may induce systematic errors which generally tend to overestimate the magnitude difference of binary stars. Hence all possible sources of systematic errors should be carefully tracked down and the whole reduction procedure extensively tested from the data acquisition to the final measurements. To do so, we set up an optical test bench experiment in the laboratory with a simulator of atmospheric turbulence, the full PISCO instrument and its ICCD detector. We could then simulate astronomical observations with a known target and then fully test our data processing method.

In Sect. 2, we present the method we used to restore images from the speckle data. The experiments on the test bench are described in Sect. 3 . In Sect. 4 we report the astronomical observations and the differential photometry measurements that have been performed on our sample of composite spectrum stars. Then in Sect. 5, we compare these measurements (i) to published data from Hipparcos and other sources, and (ii) to values computed with synthetic spectra.

\section{Presentation of the method}

\subsection{Phase retrieval and image restoration}

The phase of the Fourier transform of the object was retrieved with a bispectral method with least-square minimization as proposed by Lannes (1988). The restoration of the final image was performed with the regularized deconvolution method with support constraint in direct domain, as presented in Lannes et al. (1987a, 1987b). The whole procedure was described in detail and discussed in Prieur et al. (1991).

Both numerical experiments and full simulations on a test bench were performed, to assess the validity and robustness of the image restoration process that we had developed. In particular, these simulations have clearly shown the growing importance of a valid correction for the photon noise bias in the bispectrum when the mean number of photons per frame decreases (typically when this number is less than 500).

\subsection{Power spectrum correction for pho- ton noise bias}

In the case of low illumination, the photoelectric detection can be modeled by a Poisson process, the probability of occurrence of a single photo-event at a given location $x$ being proportional to the intensity $f(x)$ of the incident wave (Goodman 1985). We will also make the hypothesis that the ICCD detector is linear (see Sect. 3.3) and note $g(x)$, its response to a single photon. In this paper, $x$ and its dual Fourier variable $u$ are two-dimensional vectors.

Let us note $\left(d_{k}\right)$ the sequence of detected elementary frames. The intensity function $d_{k}(x)$ can then be expressed as:

$$
d_{k}(x)=\sum_{m=1}^{N_{k}} g(x) \star \delta\left(x-x_{m, k}\right),
$$

where $\star$ is the convolution operator, and $N_{k}$ is the total number of photons detected in frame $k . N_{k}$ follows Poisson's statistics with average $\bar{N}$.

With these assumptions, Dainty \& Greenaway (1979) derived the expression of the mean power spectrum of the elementary frames:

$$
E\left(|\hat{d}(u)|^{2}\right)=|\hat{g}(u)|^{2} \times\left(\bar{N}+\bar{N}^{2} \frac{|\hat{f}(u)|^{2}}{|\hat{f}(0)|^{2}}\right)
$$

Actually, Dainty \& Greenaway did not mention the photon response, i.e., they assumed $g(x)=$ $\delta(0)$.

\subsection{Bispectrum correction for photon noise bias}

At low light levels and in the case of photoncounting detection, Wirnitzer (1985) and Nakajima (1988) have shown that an unbiased estimate $\mathcal{Q}_{k}\left(\vec{\nu}_{1}, \vec{\nu}_{2}\right)$ of the bispectrum of the image can be obtained from the bispectrum $\mathcal{B}_{k}\left(\vec{\nu}_{1}, \vec{\nu}_{2}\right)$ and the Fourier transform $\hat{d}_{k}$ of the elementary frames:

$$
\begin{gathered}
\mathcal{Q}_{k}\left(\vec{\nu}_{1}, \vec{\nu}_{2}\right)=\mathcal{B}_{k}\left(\vec{\nu}_{1}, \vec{\nu}_{2}\right)-B_{p h} \\
B_{p h}=\left|\hat{d}_{k}\left(\vec{\nu}_{1}\right)\right|^{2}+\left|\hat{d}_{k}\left(\vec{\nu}_{2}\right)\right|^{2}+\left|\hat{d}_{k}\left(-\vec{\nu}_{1}-\vec{\nu}_{2}\right)\right|^{2}-2 \bar{N}
\end{gathered}
$$

where $B_{p h}$ is the bispectrum photon noise bias. Hence the photon noise bias only affects the real part of the bispectrum. In the absence of photon 
noise bias correction, the real part of the mean bispectrum is over-estimated and the amplitude of its phase is reduced (to zero, for extreme cases). Examples are given in Fig. 5.

These formulae were derived for photon-counting detectors that provide the individual photon coordinates and normalize each of them to unity, i.e., $g(x)=\delta(0)$. In the case of the ICCD detector, and with the assumptions described in Sect. 2.2, the problem can be reduced to the photon-counting case by performing a normalisation of the spectrum. Hence:

$$
\begin{gathered}
\mathcal{Q}_{k}^{i c c d}\left(\vec{\nu}_{1}, \vec{\nu}_{2}\right)=\frac{\mathcal{B}_{k}^{i c c d}\left(\vec{\nu}_{1}, \vec{\nu}_{2}\right)}{\hat{g}\left(\vec{\nu}_{1}\right) \hat{g}\left(\vec{\nu}_{2}\right) \hat{g}^{*}\left(\vec{\nu}_{1}+\vec{\nu}_{2}\right)+\epsilon}-B_{p h}^{i c c d} \\
B_{p h}^{i c c d}=\frac{\left|\hat{d}_{k}^{i c c d}\left(\vec{\nu}_{1}\right)\right|^{2}}{\left|\hat{g}\left(\vec{\nu}_{1}\right)\right|^{2}+\epsilon}+\frac{\left|\hat{d}_{k}^{i c c d}\left(\vec{\nu}_{2}\right)\right|^{2}}{\left|\hat{g}\left(\vec{\nu}_{2}\right)\right|^{2}+\epsilon}+\frac{\left|\hat{d}_{k}^{i c c d}\left(-\vec{\nu}_{1}-\vec{\nu}_{2}\right)\right|^{2}}{\left|\hat{g}\left(-\vec{\nu}_{1}-\vec{\nu}_{2}\right)\right|^{2}+\epsilon}-2 \bar{N}
\end{gathered}
$$

The $\epsilon$ (small and positive) term is added to reduce instabilities for small values of $\left|\hat{g}(u)^{2}\right|$.

The mean number of photons $\bar{N}$ per elementary frame can be determined from the values of the power spectrum at high frequency (larger than the cut frequency $u_{c}$ ).

\section{Experiments and simulations on a test bench}

We set up a laboratory simulation to check the validity and the limitations of our photometric measurements made with PISCO. This experiment allowed us to test both the data acquisition process and the whole image restoration procedure with a known target.

\subsection{Experimental setup}

The whole setup is presented in Fig. 1. The target was a photographic slide lit by a halogen lamp and placed at the focus plane of a collimating lens. Placed in the parallel beam, a slowly rotating disk (at $1 \mathrm{rpm}$ ) introduced random phase delays, to simulate the atmospheric turbulence. This disk was made of perspex with a thin layer of hair spray. The parallel beam was then re-focused to the entrance image plane of PISCO, by a set of lenses that simulated the Cassegrain focus of the TBL. The reduction scale of the pupil and the turbulence scale were chosen to simulate the conditions encountered with a two-meter aperture.

As for astronomical observations, the ICCD detector was mounted on PISCO, and data were recorded on SVHS tapes. These tapes were later processed by the same pipe-line procedure that we used for astronomical observations.

The digitization of the tapes was done by a Video
Cassette Recorder (VCR) Panasonic AG-7355 and an ELLIPS/Full RIO board. The VCR was controlled by a PC, and a program developed by JLP allowed a fully automatic digitization and processing of the SVHS tapes.

Here are the main limitations of our experiment:

- (i) the first order of turbulence is not simulated, because of the small scale of the hair spray droplets. Hence the long exposures obtained with our experiment are comparable to those obtained on the night sky with a tip-tilt correction.

- (ii) the maximum duration of a sequence is limited to one minute, because the rotation of the disk makes the turbulence periodic.

\subsection{Simulations parameters}

Among all possible setups of the ELLIPS/RIO digitizing board, we found out that the most sensitive parameters were the contrast $(\mathrm{C})$ and brightness (B). The useful range of these parameters was determined experimentally: the contrast could be tuned between $\mathrm{C}=70$ and $\mathrm{C}=90$, and the brightness had to be set accordingly between $B=140$ and $\mathrm{B}=130$, respectively. Hence, in what follows, only three setups will be considered here: $(\mathrm{C}=70$, $\mathrm{B}=140),(\mathrm{C}=80, \mathrm{~B}=130)$ and $(\mathrm{C}=90, \mathrm{~B}=130)$.

A series of simulations were performed with different configurations for various purposes. They are presented in Table 1. The simulation name and the target are given in Col. 1 and Col. 2 respectively. The atmospheric turbulence simulator could be switched on and off (Col. 3). In the following, we sometimes add G6 or G7 to the simulation name (e.g., T1-G6) according to the video gain setup for the ICCD. In those simulations, $\mathrm{VG}=6$ was selected when the target was bright, simulating bright stars; $V G=7$ was selected for faint targets.

\subsection{Linearity tests}

\subsubsection{Tests on flat disks}

For the simulations T1-G6 and T1-G7 (with video gains of $\mathrm{VG}=6$ and $\mathrm{VG}=7$ respectively), the target was a slide with four "flat disks", constituted by frosted circular areas. The brightness ratio between the brightest (D1) and the faintest (D4) disks was very large $(\Delta m \sim 3.4)$ so that this linearity test could cover a large dynamical range. We used a set of neutral density attenuators whose transmission varied from 1 . to $10^{-2}$, to lit this target with a wide range of illumination (ratio of 100). 


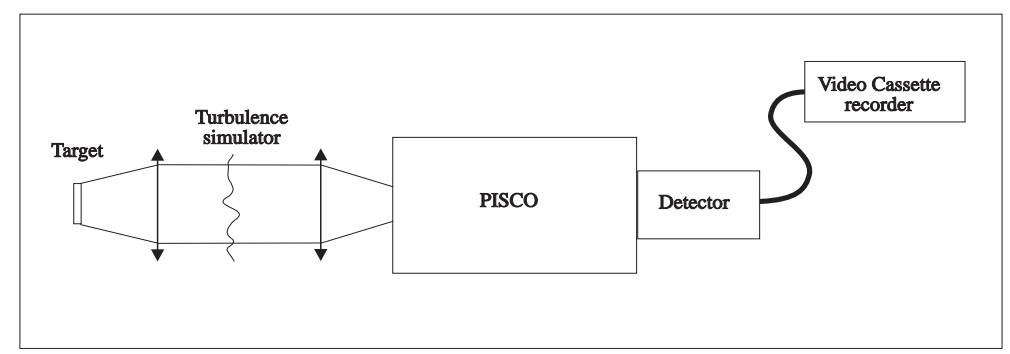

Fig. 1.- Simulations on the test bench: experimental setup

TABLE 1

Simulations ON THE TEST BENCH.

\begin{tabular}{cccc}
\hline \hline Name & Target & Turbulence & Purpose \\
& & & \\
\hline T1 & Flat disks & no & Linearity tests \\
T2 & Double star & no & Linearity tests \\
S1 & Double star & yes & Image restoration \\
S2 & Single star & no & Photon noise bias \\
\hline
\end{tabular}

Photometric measurements of the four disks were obtained on "long exposures", i.e., by integrating the elementary frames during about 1 minute. They are plotted in Fig. 2 versus the measurements of T1-G6, used as a reference and corresponding to a good illumination and a small video gain $(\mathrm{VG}=6)$. This figure shows that the photometric response of the system (ICCD detector + digitizing board) can be considered as linear. The mean departure from linearity is $3 \%$ for the high levels (disk D2, $\Delta m=0.08$ ), $5 \%$ for the low levels (disk D3, $\Delta m=1.58$ ), and $8 \%$ for the very low levels (disk D4, $\Delta m=3.44$ ). That response is not very sensitive to the illumination of the target within the wide domain (ratio 100) that we have explored. During this experiment, the best setup for the digitization parameters was found to be $(\mathrm{C}=80, \mathrm{~B}=130)$ for $\mathrm{VG}=7$ and $(\mathrm{C}=70, \mathrm{~B}=140)$ for $\mathrm{VG}=6$.

\subsubsection{Tests with point-like sources}

Still images of simulated double stars without turbulence, were integrated during $\sim 30 \mathrm{~s}$. The resulting measurements showed that relative photometry of point-like sources is not reliable when the stellar images are well focused and concentrated to only a few pixels. This is caused by the saturation of the channels of the micro-channel plate used in front of the CCD. To reduce this effect, it is necessary to defocus the image to spread it over a large number of pixels (like in the case of "flat disks"). This condition is fortunately often verified during astronomical observations, since (i) a large magnification is used to correctly sample the Airy disk, and (ii) the atmospheric turbulence makes the image wander over the image.

\subsection{Photon noise bias and ICCD photon response $g(x)$}

We performed a series of experiments with low illumination to study both the ICCD photon response and the effects of the photon noise bias on the mean power spectrum and mean bispectrum of the elementary frames.

\subsubsection{Photon noise bias on the power spectrum}

To evaluate the value of the power spectrum $F^{2}(u) \stackrel{\text { def }}{=}|\hat{f}(u)|^{2}$, of a steady brightness distribution $f(x)$ that lights the surface of a detector, one would naturally think to use $D^{2}(u) \stackrel{\text { def }}{=}$ $E\left(|\hat{d}(u)|^{2}\right)$, the average of the power spectra of the detected elementary frames $d(x)$. As shown in Sect. 2.2, this estimator is biased for low illumination levels. Likewise, its bispectrum $F^{3}\left(u_{1}, u_{2}\right) \stackrel{\text { def }}{=} \hat{f}\left(u_{1}\right) \hat{f}\left(u_{2}\right) \hat{f}^{*}\left(u_{1}+u_{2}\right)$ cannot be approximated by the average bispectrum $D^{3}(u) \stackrel{\text { def }}{=} E\left(\hat{d}\left(u_{1}\right) \hat{d}\left(u_{2}\right) \hat{d}^{*}\left(u_{1}+u_{2}\right)\right)$.

During experiment $S 2$, an image of a low-illuminated steady target without turbulence simulator was projected to the ICCD entrance faceplate. This target was chosen as a point-like object, and its image was slightly de-focused. The result- 


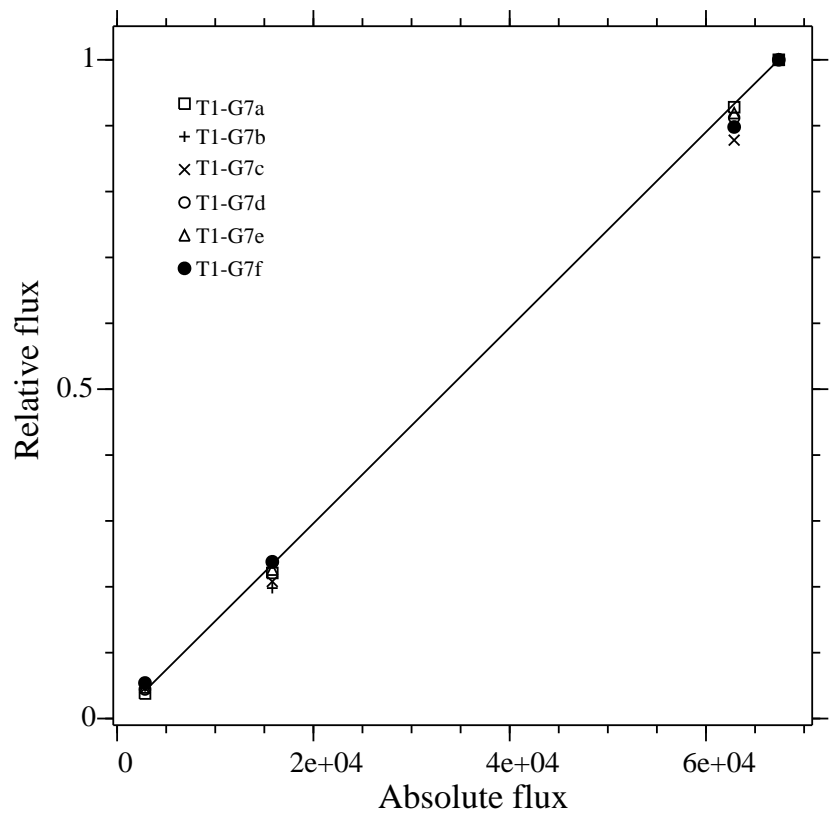

Fig. 2.- Linearity tests with experiments T1-G7a to T1-G7f. Solid line corresponds to the ideal linear case. The "absolute" flux of each disk was measured with $\mathrm{VG}=6$ with a good illumination of the target.

ing mean power spectrum $D^{2}(u)$ of the elementary frames is plotted in Fig. 3. Compared to the power spectrum $D_{0}^{2}(u)$ of the average frame $d_{0}(x)=E(d(x))$, we note (i) the presence of a nonuniform background and (ii) a bright peak at zero frequency. Hence, $D^{2}(u)$ is biased (cf. Sect. 2.2). The existence of a non-zero background in $D^{2}(u)$ (Fig. 3) can be explained by the "photon noise bias" $|\hat{g}(u)|^{2} \times \bar{N}$. The non-uniformity of this background (as would be expected for a perfect photon-counting detector) is an indication that $g(x)$ is not concentrated to a single pixel.

The horizontal pattern of $g(x)$ with a negative lobe is due to losses during the transfer of charges across the CCD. By construction, we found a symmetric pattern since $g(x)$ was derived it from its power spectrum. As shown by Germain \& Douglass (2001) in their Fig. 2, the actual ICCD photon response is asymmetric. As charge packets are clocked across the CCD, traps tend to be filled by electrons from the leading pixels within a speckle. For adjacent pixels, the charge on the pixel closer to the CCD output is then systematically lowered relative to the trailing pixel.

\subsubsection{Determination of the ICCD photon re- sponse $g(x)$}

We measured the power spectrum $|\hat{g}(u)|^{2}$ from a series of frames of a flat field with a low illumination, and $\mathrm{VG}=7$ (cf. Fig. 4). In this case, $\hat{f}(u)=\delta(u)$. Hence $E\left(|\hat{d}(u)|^{2}\right)=|\hat{g}(u)|^{2} \times \bar{N}$ for all non-zero frequencies (Cf. Eq. 2).

To determine $\hat{g}(u)$, the value of $\bar{N}$, the mean number of photons per frame, was needed. We then performed two simulations S3: (S3-a) with a moderately low illumination to determine the relative value of $|\hat{g}(u)|^{2}$ with a good SNR, and (S3-b) with a very low illumination so that photons could be clearly identified and counted on elementary frames. From the value $\bar{N}$ measured for (S3-b) and the ratio of the power spectra obtained the simulations S3-a and S3-b, we could derive the value of $\bar{N}$ for(S3-a).

During this experiment, we noted that the level of the response function to a single photon was not constant (absolute variation of about 30\%). The assumption of a convolution by a fixed function $g(x)$ is only an approximation. Nevertheless, with this determination of $g(x)$, the correction of the experimental power spectrum as described in Sect. 2.2 was satisfactory as shown in Fig. 3. In next section, we will see that the correction of the bispectrum with $g(x)$ and the procedure described in Sect. 2.3 also led to correct photometric measurements.

\subsection{Photometric measurements of simu- lated double stars with turbulence}

To check the reliability of photometric measurements of a binary object in the presence of atmospheric turbulence, simulation (S1) was thus performed with different values for the turbulence strength and a wide range of illumination. We present here four data sequences extracted from the SVHS recordings, with $\mathrm{VG}=6$ and designed as: S1-G6-a, S1-G6-b, S1-G6-c and S1-G6d (cf. Fig. 5). The SNR of the maxima of the corresponding "long exposure", obtained by integrating the elementary frames, were 19, 2.0, 1.2 and 0.9 respectively, with a FWHM seeing of 6 , 16, 18 and 17 pixels respectively.

Photometric measurements derived from simulation S1 are given in Table 2. The measurements performed on the long exposures (which are equivalent to tip-tilt corrected long exposures, as already mentioned in Sect. 3) are given in Col. 2. Those obtained on the restored images using the bispectral method described in Sect. 2 are given in Cols. 3 and 4. The "raw" values (Col. 3) are relative to images obtained without correction, whereas the "corrected" values (Col. 4) correspond to images whose phase was restored after photon noise bias correction with the range of $\bar{N}$ values given in Col. 5 (i.e., mean number of photons per frame). As the components of this double star were well separated (cf. Fig. 5), the intensity ra- 
tio could be directly measured on the images restored after phase retrieval only, without any need for deconvolution.

As explained in Sect. 2.3, the value of $\bar{N}$ is determined from the values of the power spectrum at high frequency. As it is not strictly constant after the cut frequency, a whole range of values are possible for $\bar{N}$ (Col. 5). We then computed the mean $\Delta m$ obtained within that range, but it appeared that the photometry is not very sensitive to the value of $\bar{N}$ (i.e. less than 0.05 magnitudes).

In the simulation $\mathrm{S} 1$, the expected value for $\Delta m$ was $0.07 \pm 0.01$, as measured with long exposures with a good illumination (S1-G6a, b and c). Table 2 shows clearly that, for obtaining a good photometric measurement, it is essential to perform a correction for the photon noise bias and for the detector photon response for both the power spectrum and the mean bispectrum (cf. Sect. 2.2 and 2.3).

During these simulations, phase restoration proved crucial for obtaining good photometry. Even when a strongly degraded modulus was used, the restored image led to correct results as soon as the phase was good. This simulation also gives an indication of the accuracy that we can obtain with PISCO and the ICCD: the mean value of Col. 4 is $\langle\Delta m\rangle=0.09 \pm 0.03$ magnitude.

\section{Photometry of binary stars}

\subsection{Observations}

The observations were made with PISCO $^{1}$ installed at the Cassegrain focus of the 2-meter telescope at Pic du Midi during the period 1993-1998. As these observations were thoroughly described in Paper I, we simply describe here the filter system that we have used (Cf. Table 3 and Fig. 6). The observations presented here were performed with two detectors: the CAR (Caméra à Anode Résistive or Ranicon-type detector), of Observatoire de la Côte d'Azur (OCA) and the ICCD (Intensified CCD with a micro-channel plate) of Université de Nice.

\subsection{Photometric measurements}

We restored high resolution images with the bispectrum technique as described in Prieur et al. (1991) and derived the differential photometry of the two components.

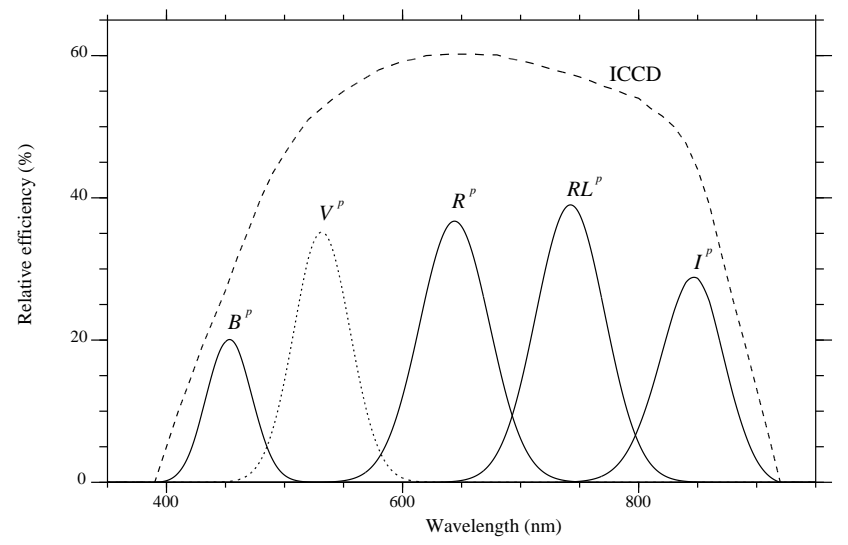

Fig. 6.- Relative efficiency curve of PISCO filters combined with the ICCD response (upper curve in dashed line). 
TABLE 2

Photometric MEASUREMENTS OBTAINED FROM THE SiMUlation S1, With DIFFERENT TURBULENCE STRENGTH.

\begin{tabular}{ccccc}
\hline \hline $\begin{array}{c}\text { Name } \\
(1)\end{array}$ & $\begin{array}{c}\Delta m \text { (long) } \\
(2)\end{array}$ & $\begin{array}{c}\Delta m \text { (raw) } \\
(3)\end{array}$ & $\begin{array}{c}\Delta m \text { (corr.) } \\
(4)\end{array}$ & $\begin{array}{c}\bar{N} \text { (phot) } \\
(5)\end{array}$ \\
\hline S1-G6-a & 0.06 & 0.34 & 0.12 & $80-300$ \\
S1-G6-b & 0.06 & 0.84 & 0.05 & $150-500$ \\
S1-G6-c & 0.08 & 0.44 & 0.09 & $200-400$ \\
S1-G6-d & 0.11 & 0.81 & 0.05 & $200-300$ \\
S1-G7bb & -0.07 & 2.04 & 0.08 & $30-60$ \\
S1-G7cc & 0.01 & 0.40 & 0.09 & $30-150$ \\
S1-G7gg & 0.14 & 1.23 & 0.14 & $30-60$ \\
\hline
\end{tabular}

TABLE 3

FILTER CHARACTERISTICS.

\begin{tabular}{ccc}
\hline \hline Name & $\begin{array}{c}\text { Central wavelength } \\
(\mathrm{nm})\end{array}$ & $\begin{array}{c}\text { Bandwidth } \\
(\mathrm{nm})\end{array}$ \\
\hline$B^{P}$ & 447 & 47 \\
$V^{P}$ & 530 & 57 \\
$R^{P}$ & 644 & 70 \\
\hline
\end{tabular}


TABle 4

Photometric measurements With PISCO: Composite SPECTRUm STARS

\begin{tabular}{|c|c|c|c|c|c|c|c|c|c|c|}
\hline $\begin{array}{l}\text { Ident. } \\
\text { (1) }\end{array}$ & $\begin{array}{l}\text { Model } \\
(2)\end{array}$ & $\begin{array}{c}B-V \\
(3)\end{array}$ & $\begin{array}{c}\Delta B \\
(4)\end{array}$ & $\begin{array}{c}\Delta B^{P} \\
(5)\end{array}$ & $\begin{array}{c}\Delta V^{P} \\
(6)\end{array}$ & $\begin{array}{l}\Delta V \\
(7)\end{array}$ & $\begin{array}{c}\Delta R^{P} \\
\quad(8)\end{array}$ & $\begin{array}{c}\Delta R \\
(9)\end{array}$ & $\begin{array}{c}\text { Epoch } \\
(10)\end{array}$ & $\begin{array}{c}\text { Comments } \\
\text { (11) }\end{array}$ \\
\hline HD 8036 & & 0.64 & & $-0.32 \pm .1$ & $0.65 \pm .1$ & & $1.68 \pm .1$ & & 1998.66 & $\rho=1.6^{\prime \prime}$ \\
\hline HIP 6226 & & & $-0.20 \pm .15$ & & & $0.57 \pm .15$ & & $\cdots$ & 1991.25 & FAB2000a \\
\hline \multirow[t]{5}{*}{ 01198-0031 } & & & $\ldots$ & & & 0.52 & & $\ldots$ & 1991.25 & HIP1997a \\
\hline & & & $\ldots$ & & & 0.54 & & $\ldots$ & 1991.25 & TYC2000a \\
\hline & & & -0.24 & & & 0.57 & & $\cdots$ & & GIN2002 \\
\hline & $G 9 I I I-I V+A 7 I V$ & 0.58 & -0.15 & 0.02 & 0.52 & 0.60 & 0.98 & 0.98 & & $1.1,1.7$ \\
\hline & $K 2 I I I-I V+A 7 I V$ & 0.65 & -0.30 & -0.11 & 0.50 & 0.60 & 1.09 & 1.08 & & $1.1,1.7$ \\
\hline HD 12447 & & 0.03 & & $1.68 \pm .1$ & $0.94 \pm .1$ & & $1.03 \pm .1$ & & 1998.66 & $\rho=1.9^{\prime \prime}$ \\
\hline HIP 9487 & & & & $1.68 \pm .1$ & $\ldots$ & & $1.07 \pm .1$ & & 1998.66 & \\
\hline \multirow[t]{4}{*}{$02020+0246 \mathrm{AB}$} & & & $1.08 \pm .15$ & & & $1.02 \pm .15$ & & $\cdots$ & 1991.25 & FAB2000a \\
\hline & & & $\ldots$ & & & 1.10 & & $\ldots$ & 1991.25 & HIP1997a \\
\hline & & & $\ldots$ & & & 1.07 & & $\cdots$ & 1991.25 & TYC2000a \\
\hline & & & $\cdots$ & & & 0.9 & & $\cdots$ & 1995.7 & WSI1997 \\
\hline \multirow{4}{*}{$\begin{array}{l}\text { HD } 18925 \\
\text { HIP } 14328 \\
03048+5330\end{array}$} & & 0.72 & & $0.33 \pm .1$ & $0.98 \pm .2$ & & $1.15 \pm .1$ & & 1998.66 & $\rho=0.3^{\prime \prime}$ \\
\hline & & & $\ldots$ & & & $\cdots$ & & $1.5 \pm .5$ & 1974. & LAB1974 \\
\hline & & & $\cdots$ & & & 1.3 & & $\ldots$ & & GIN2002 \\
\hline & G9II-III+A2III & 0.61 & 0.23 & 0.43 & 1.00 & 1.10 & 1.58 & 1.57 & & $-1.0,0.1$ \\
\hline HD 29140 & & & & $1.7 \pm .2$ & $1.6 \pm .2$ & & $1.0 \pm .1$ & & 1997.07 & $\rho=0.2^{\prime \prime}$ \\
\hline HIP 21402 & & & $\ldots$ & & & $\ldots$ & & $1.85 \pm .15$ & 1994.7 & SCO1998 \\
\hline \multicolumn{11}{|l|}{$04357+1010$} \\
\hline HD 74874 & & 0.69 & & $\cdots$ & $0.9 \pm .2$ & & $\cdots$ & & 1997.07 & $\rho=0.3^{\prime \prime}$ ARI1999 \\
\hline HIP 43109 & & & $\ldots$ & & & 1.2 & & $\ldots$ & 1988 & ISM1992 \\
\hline \multirow[t]{3}{*}{$08468+0625 \mathrm{AB}$} & & & $\cdots$ & & & $1.0 \pm 0.15$ & & $\cdots$ & 1997 & HOR2001a \\
\hline & & & $\cdots$ & & & 0.75 & & $\cdots$ & & GIN2002 \\
\hline & $G 7 I I I+A 8 I I I-I V$ & 0.63 & 0.18 & 0.35 & 0.82 & 0.90 & 1.25 & 1.25 & & $0.8,1.7$ \\
\hline HD 166479 & & 0.57 & & $\cdots$ & $0.80 \pm .1$ & & $1.65 \pm .1$ & & 1998.66 & $\rho=1.2^{\prime \prime}$ \\
\hline HIP 89023 & & & $-0.72 \pm .15$ & & & $0.53 \pm .15$ & & $\cdots$ & 1991.25 & FAB2000a \\
\hline \multirow[t]{6}{*}{$18101+1629$} & & & $\ldots$ & & & 0.43 & & $\cdots$ & 1991.25 & HIP1997a \\
\hline & & & $\cdots$ & & & 0.56 & & $\cdots$ & 1991.25 & TYC2000a \\
\hline & & & $\ldots$ & & & 1.1 & & $\ldots$ & 1991 & WSI1997 \\
\hline & & & $\cdots$ & & & 0.8 & & $\cdots$ & 1993 & WSI1997 \\
\hline & & & $\ldots$ & & & 0.54 & & $\cdots$ & & GIN2002 \\
\hline & $G 8 I I-I I I+B 9 V$ & 0.51 & -0.09 & 0.14 & 0.80 & 0.90 & 1.40 & 1.39 & & $-0.7,0.2$ \\
\hline HD 183912 & & 1.09 & & $1.16 \pm .1$ & $1.75 \pm .1$ & & $2.10 \pm .3$ & & 1998.66 & $\rho=0.4^{\prime \prime}$ \\
\hline HIP 95947 & & & $0.58 \pm .15$ & & & $2.15 \pm .15$ & & $\ldots$ & 1991.25 & FAB2000a \\
\hline \multirow[t]{5}{*}{$19307+2758 \mathrm{Aa}$} & & & $\ldots$ & & & 2.12 & & $\ldots$ & 1991.25 & HIP1997a \\
\hline & & & $\ldots$ & & & 1.9 & & $\ldots$ & 1991.25 & TYC2002 \\
\hline & & & $\cdots$ & & & $2.64 \pm .19$ & & $3.47 \pm .26$ & 1996. & TТВ2000 \\
\hline & & & $\cdots$ & & & 2.2 & & $\ldots$ & & GIN2002 \\
\hline & $K 2 I I+B 8 V$ & 0.78 & 0.69 & 0.98 & 1.77 & 1.90 & 2.53 & 2.52 & & $-2.3,-0.4$ \\
\hline HD 186203 & & 0.58 & & $\cdots$ & $1.00 \pm .1$ & & $1.49 \pm .1$ & & 1998.66 & $\rho=0.4^{\prime \prime}$ \\
\hline HIP 96957 & & & $-0.16 \pm .15$ & & & $1.03 \pm .15$ & & $\cdots$ & 1991.25 & FAB2000a \\
\hline \multirow[t]{6}{*}{$19426+1150$} & & & $\cdots$ & & & 0.88 & & $\cdots$ & 1991.25 & HIP1997a \\
\hline & & & $\ldots$ & & & 1.0 & & $\ldots$ & 1991.25 & TYC2002 \\
\hline & & & $\ldots$ & & & 1.2 & & $\cdots$ & 1992.7 & WSI1997 \\
\hline & & & $\ldots$ & & & 1.1 & & $\ldots$ & $1994 ?$ & WSI1999b \\
\hline & & & $\ldots$ & & & 1.1 & & $\ldots$ & & GIN2002 \\
\hline & $G 2 I b-I I+B 5 V$ & 0.49 & 0.06 & 0.30 & 0.99 & 1.10 & 1.60 & 1.59 & & $-2.3,-1.2$ \\
\hline HD 186518 & & 1.02 & & $\cdots$ & $\cdots$ & & $1.79 \pm .2$ & & 1998.66 & $\rho=0.3^{\prime \prime}$ \\
\hline HIP 97091 & & & $-0.78 \pm .15$ & & & $1.28 \pm .15$ & & $\cdots$ & 1991.25 & FAB2000a \\
\hline \multirow[t]{3}{*}{$19441+2708$} & & & $\cdots$ & & & 1.20 & & $\cdots$ & 1991.25 & HIP1997a \\
\hline & & & $\ldots$ & & & 1.2 & & $\ldots$ & & GIN2002 \\
\hline & $K 3 I I+B 6 V$ & 0.65 & -0.15 & 0.18 & 1.05 & 1.20 & 1.90 & 1.89 & & $-2.2,-1$ \\
\hline HD 187259 & & 0.55 & & $0.18 \pm .1$ & $0.31 \pm .1$ & & $0.51 \pm .1$ & & 1998.66 & $\rho=1.5^{\prime \prime}$ \\
\hline HIP 97473 & & & & $\ldots$ & $0.32 \pm .1$ & & $\ldots$ & & 1998.66 & \\
\hline $19487+1149$ & & & $-0.64 \pm .15$ & & & $0.39 \pm .15$ & & $\cdots$ & 1991.25 & FAB2000a \\
\hline & & & $\ldots$ & & & 0.29 & & $\ldots$ & 1991.25 & HIP1997a \\
\hline & & & $\ldots$ & & & 0.41 & & $\ldots$ & 1991.25 & TYC2000a \\
\hline & & & $\ldots$ & & & 0.8 & & $\ldots$ & 1993 & WSI1999a \\
\hline
\end{tabular}



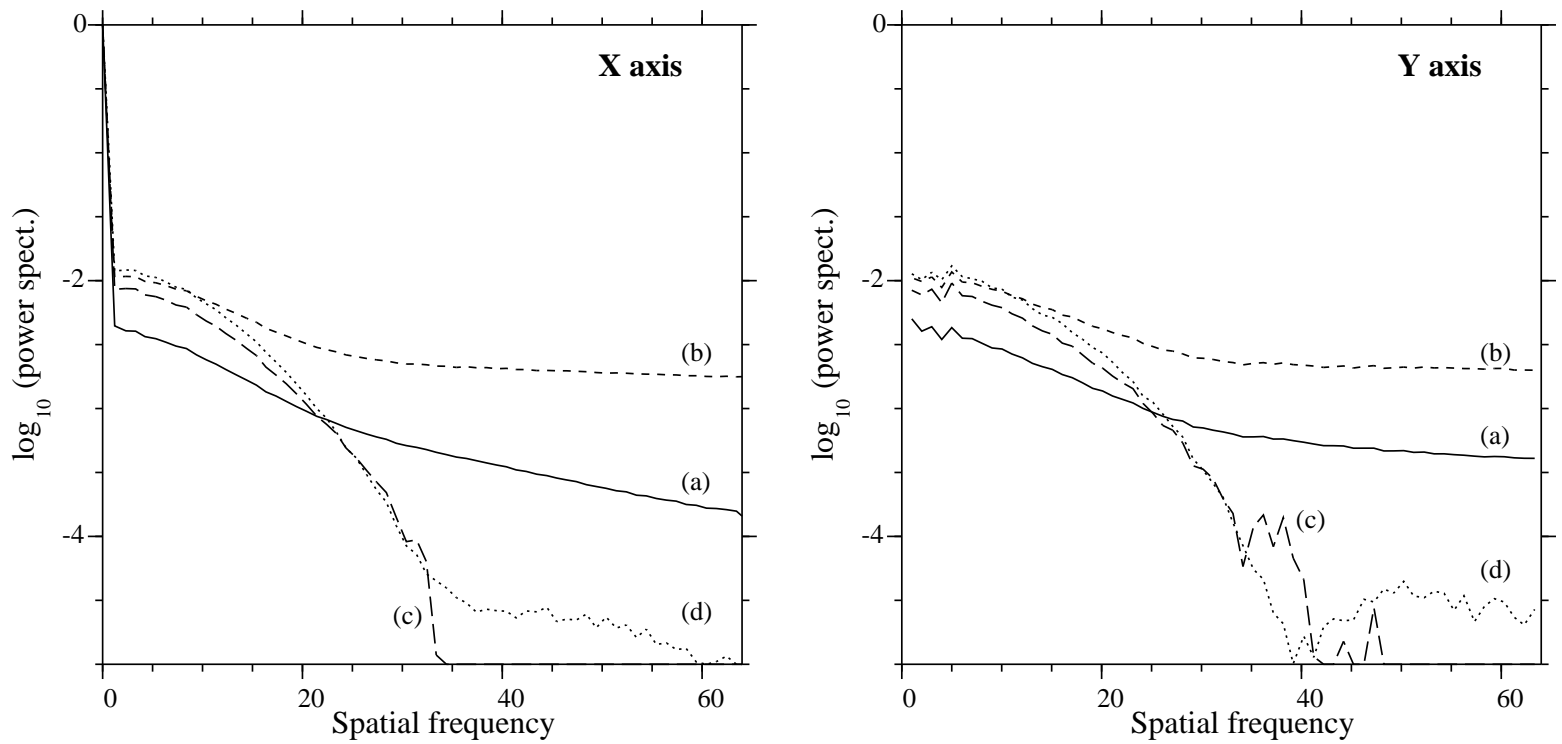

Fig. 3.- Photon noise bias with ICCD: normalized mean power spectrum of elementary frames obtained with experiment S2: raw (a), corrected for photon response (b), corrected for photon response and photon noise bias (c). Unbiased power spectrum (d) from a high illumination exposure. Left: cut along the X axis, right: cut along the $\mathrm{Y}$ axis.

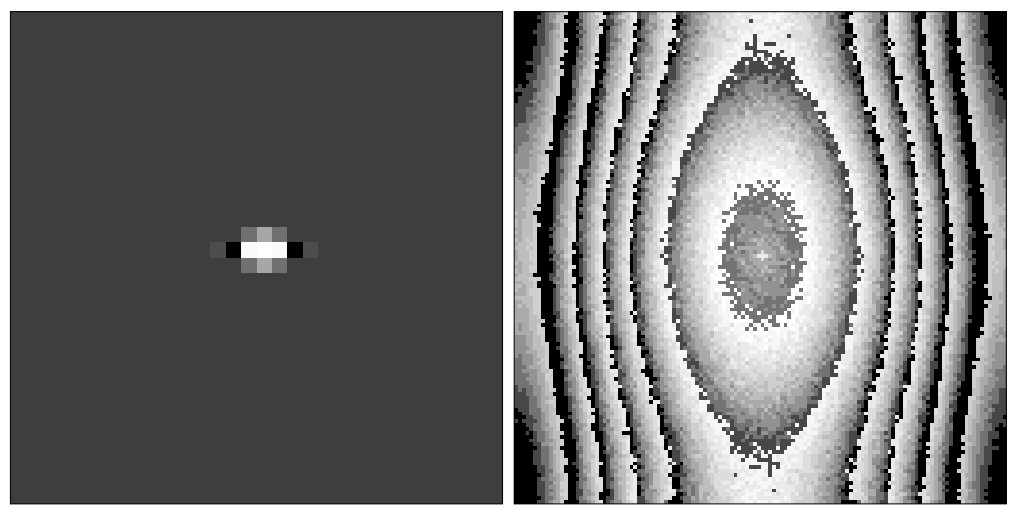

Fig. 4.- ICCD response function $g(x)$ to a single photo-event (left) and corresponding power spectrum (right). 


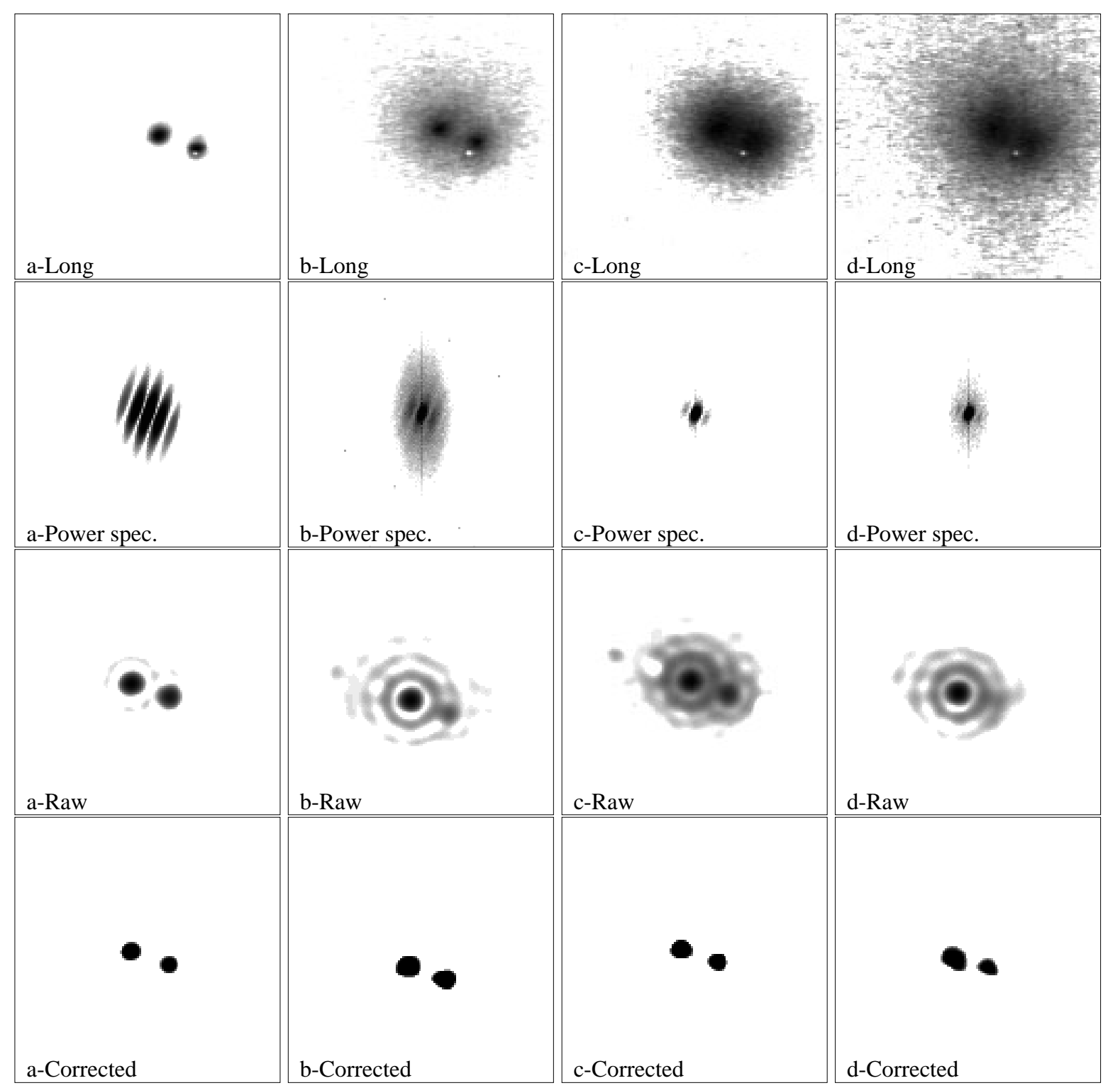

Fig. 5.- Simulation S1, with different strength of turbulence, increasing from left to right: S1-G6-a, S1-G6b, S1-G6-c, S1-G6-d. From top to bottom: long exposure, mean power spectrum, images whose phase was restored from the raw bispectrum, restored images using bispectrum and power spectrum both corrected for the photon noise bias and detector photon response. 
The photometric measurements are displayed in Tables 4 and 5. For each observation, we give the name of the object (HD or WDS in col. 1), the global color index $B-V$ of the binary system (in col. 3) measured by Hipparcos (ESA 1997), and the relative photometry $\Delta B^{P}, \Delta V^{P}$, and $\Delta R^{P}$ (Cols. 5, 6 and 8) relative to the filter system described in Table 3, and when available the photometry $\Delta B, \Delta V$, and $\Delta R$ (Cols. 4, 7, and 9 ) given by other sources. The code used for those sources is the same as in the Fourth Catalog of Interferometric Measurements of Binary Stars (Hartkopf et al., 2002): ARI1999 for Aristidi et al. (1999), FAB2000a for Fabricius \& Makarov (2000), HIP1997a for ESA (1997), HOR2002 for Horch et al. (2002), ISM1992 for Ismailov (1992), LAB1974 for Labeyrie et al. (1974), SCO1998 for Schoeller et al. (1998), TTB2000 for ten Brummelaar et al. (2000), TYC2000a for Hog et al. (2000), TYC2002 for Fabricius et al. (2002), WSI1997 for Douglass et al. (1997), WSI1999a for Germain et al. (1999a), WSI1999b for Germain et al. (1999b), and WSI2000a for Douglass et al. (2000). We added the code GIN2002 for Ginestet \& Carquillat (2002). The epoch of observation in Besselian years is in col. 10, and comments in col. 11.

To compare our photometric measurements with theoretical values that can be derived from models, we have used Pickles' spectral library HILIB (Pickles 1998). We developed a program that could compute, for two stars of any spectral type, the expected output flux for a given filter using the efficiency curves (filter + ICCD response) of Fig. 6 (e.g., Fig. 7). In Table 4, we give (in slanted fonts) the relative photometry corresponding to the combination of the spectral types given in Col. 2. Unless explicitely stated, the spectral types were taken from GIN2002. In the same line, the $B-V$ in Col. 3 is derived from this composite spectrum. Absolute visual magnitudes were determined for each component (same line, Col. 11), so that they lead to a satisfactory agreement with the observations (see discussion in Sect. 5.2).

In this table, the measurements from other sources are "raw" values (i.e., not converted to Johnson's system).

Note that the indices $B-V$ measured by Hipparcos are affected by the interstellar redening, while those computed by spectral models are "intrinsic". One can check in Col. 3 that the latter are smaller than the former, as they should actually be. Note also that our differential measurements are not sensitive to interstellar redening.

\subsection{Individual cases (Table 4)}

HD 8036: GIN2002 gives $G 9 I I I-I V+A 7 I V$

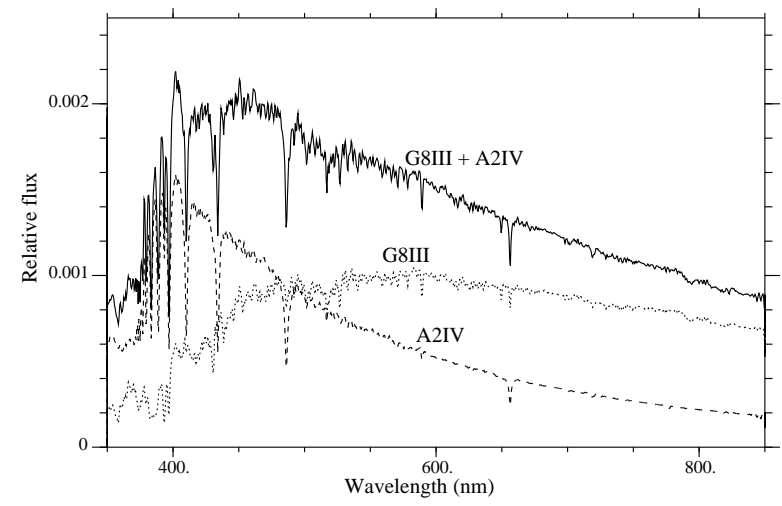

Fig. 7.- Example of synthetic spectrum used for HD187259: G8III + A2IV, with absolute visual magnitudes of 0.8 and 1.2 respectively.

but K2III + A7IV better agrees the PISCO data. It was also classified as G8III: + A7V (Bidelman 1958) and KIII: + A7V (Cowley 1976). $d=121$ pc. HD 12447: $\alpha$ Psc, is a well known orbital visual binary (Scardia 1983). Both components are chemically peculiar hot stars: the primary is A0p, and the secondary is Am; $\Delta m_{V}=1$. According to Bright Star Catalogue (Hoffleit \& Jaschek 1982), both components of the visual binary are possibly spectroscopic binary systems. On the long exposure frames, the two stars are marginally separated: the intensity of the secondary is much lower in $B$ than in $V$, and the contrast between the two components is of the same order in $V$ and in $R$. This is a good agreement with our measurements obtained on the restored images. Therefore, the value $\Delta B=1.08$ given by FAB2000a is dubious.

HD 18925: $\gamma$ Per, is a well known eclipsing, spectroscopic and interferometric system (see Batten et al. 1989, Popper \& McAlister 1987, Pourbaix 1999). The absolute magnitudes we find for the two components (Col. 11) are slightly different from the values given by GIN2002 $\left(M_{V 1}=-1.25\right.$ and $M_{V 2}=0.05$ ), for the same classification G9IIIII + A2III. $d=79$ pc.

HD 29140: 88 Tau. The primary is a spectroscopic binary of Am type $\left(P=3.57\right.$ days, $\Delta m_{V}=$ 2.31). A red secondary spectrum was detected by Fekel (Batten et al 1989). According to Tokovinin \& Gorynya (2001), this is a sextuple system!

HD 74874: $\epsilon$ Hya, is a variable star of BY Dra type, both orbital spectroscopic and interferometric binary (see Batten et al. 1989 and Hartkopf et al. 1996). According to Batten et al., the system is at least quintuple. Bright Star Catalogue gives $\Delta m_{V}=0.9 . d=41 \mathrm{pc}$.

HD 166479: Bright Star Catalogue gives $\Delta m_{V}=$ 0.8. GIN2002 gives G8III + B9.5V. $d=461 \mathrm{pc}$, but 
TABLE 4-Continued

\begin{tabular}{|c|c|c|c|c|c|c|c|c|c|c|}
\hline $\begin{array}{l}\text { Ident. } \\
(1)\end{array}$ & $\begin{array}{c}\text { Model } \\
(2)\end{array}$ & $\begin{array}{c}B-V \\
(3)\end{array}$ & $\begin{array}{c}\Delta B \\
(4)\end{array}$ & $\begin{array}{c}\Delta B^{P} \\
(5)\end{array}$ & $\begin{array}{c}\Delta V^{P} \\
(6)\end{array}$ & $\begin{array}{l}\Delta V \\
(7)\end{array}$ & $\begin{array}{c}\Delta R^{P} \\
(8)\end{array}$ & $\begin{array}{c}\Delta R \\
(9)\end{array}$ & $\begin{array}{c}\text { Epoch } \\
(10)\end{array}$ & $\begin{array}{c}\text { Comments } \\
\text { (11) }\end{array}$ \\
\hline & $G 8 I I I+A 2 I V$ & 0.49 & $\begin{array}{c}\cdots \\
-0.45\end{array}$ & -0.25 & 0.31 & $\begin{array}{c}0.4 \\
0.40\end{array}$ & 0.85 & $\begin{array}{l}\ldots \\
0.84\end{array}$ & & $\begin{array}{l}\text { GIN2002 } \\
0.4,0.8\end{array}$ \\
\hline $\begin{array}{l}\text { HD } 187321 \\
\text { HIP } 97476 \\
19487+1852\end{array}$ & $G 5 I I+B 7 I V$ & 0.86 & $\begin{array}{c}0.17 \pm .15 \\
\ldots \\
\ldots \\
\ldots \\
0.38\end{array}$ & $\cdots$ & $1.34 \pm .2$ & $\begin{array}{c}1.61 \pm .15 \\
1.46 \\
1.7 \\
1.5 \\
1.40\end{array}$ & $\begin{array}{c}1.80 \pm .2 \\
1.88\end{array}$ & $\begin{array}{l}\ldots \\
\ldots \\
\ldots \\
\ldots \\
1.88\end{array}$ & $\begin{array}{l}1998.66 \\
1991.25 \\
1991.25 \\
1991.25\end{array}$ & $\begin{array}{l}\rho=0.4^{\prime \prime} \\
\text { FAB2000a } \\
\text { HIP1997a } \\
\text { TYC2002 } \\
\text { GIN2002 } \\
-2.5,1.1\end{array}$ \\
\hline $\begin{array}{l}\text { HD } 194359 \\
\text { HIP } 100643 \\
20244+2417\end{array}$ & $G 8 I I I+A 1 V$ & 0.71 & $\begin{array}{c}0.47 \pm .15 \\
\ldots \\
\ldots \\
\ldots \\
\ldots \\
0.27\end{array}$ & 0.47 & $1.09 \pm .2$ & $\begin{array}{c}1.5 \pm .15 \\
1.39 \\
0.5 \\
0.9 \\
1.2 \\
1.20\end{array}$ & $1.78 \pm .3$ & $\begin{array}{l}\ldots \\
\ldots \\
\ldots \\
\ldots \\
\ldots \\
1.70\end{array}$ & $\begin{array}{l}1998.66 \\
1991.25 \\
1991.25 \\
1991.25 \\
1994 .\end{array}$ & $\begin{array}{l}\rho=0.3^{\prime \prime} \\
\text { FAB2000a } \\
\text { HIP } 1997 \mathrm{a} \\
\text { TYC2002 } \\
\text { WSI1999a } \\
\text { GIN2002 } \\
-0.2,1.0\end{array}$ \\
\hline $\begin{array}{l}\text { HD } 195692 \\
\text { HIP } 101300 \\
20320+2548\end{array}$ & & & $\ldots$ & $\cdots$ & $\cdots$ & 2.17 & $0.38 \pm .1$ & $\ldots$ & $\begin{array}{l}1998.66 \\
1991.25\end{array}$ & $\begin{array}{l}\rho=0.2^{\prime \prime} \\
\text { HIP } 1997 \mathrm{a}\end{array}$ \\
\hline $\begin{array}{l}\text { HD } 215242 \\
\text { HIP } 112170 \\
22431+4710\end{array}$ & $G 8 I I-I I I+B 7 I V$ & 0.45 & $\begin{array}{c}-1.32 \pm .15 \\
\ldots \\
\ldots \\
\ldots \\
-1.36\end{array}$ & -1.11 & -0.41 & $\begin{array}{c}0.04 \pm .15 \\
0.07 \\
\ldots \\
-0.3 \\
-0.30\end{array}$ & $0.10 \pm .1$ & $\begin{array}{c}\ldots \\
\ldots \\
1.43 \pm 0.08 \\
\ldots \\
0.22\end{array}$ & $\begin{array}{l}1998.66 \\
1991.25 \\
1991.25 \\
1991.25\end{array}$ & $\begin{array}{l}\rho=0.5^{\prime \prime} \\
\text { FAB2000a } \\
\text { HIP1997a } \\
\text { SCO1998 } \\
\text { GIN2002 } \\
-0.6,-0.9\end{array}$ \\
\hline
\end{tabular}

TABLE 5

Photometric measurements with PISCO: COMPlementary list

\begin{tabular}{|c|c|c|c|c|c|c|c|c|}
\hline $\begin{array}{l}\text { Ident. } \\
(1)\end{array}$ & $\begin{array}{c}\Delta B \\
(4)\end{array}$ & $\begin{array}{c}\Delta B^{P} \\
(5)\end{array}$ & $\begin{array}{c}\Delta V^{P} \\
(6)\end{array}$ & $\begin{array}{c}\Delta V \\
(7)\end{array}$ & $\begin{array}{c}\Delta R^{P} \\
(8)\end{array}$ & $\begin{array}{l}\Delta R \\
(9)\end{array}$ & $\begin{array}{c}\text { Epoch } \\
(10)\end{array}$ & $\begin{array}{c}\text { Comments } \\
(11)\end{array}$ \\
\hline HD 74874 & & $\ldots$ & $1.9 \pm .2$ & & $\ldots$ & & 1997.07 & $\rho=2.9^{\prime \prime}$, ARI1999 \\
\hline HIP 43109 & $\ldots$ & & & 3.10 & & $\ldots$ & 1991.25 & HIP 1997a \\
\hline $08468+0625 \mathrm{AC}$ & $\cdots$ & & & 2.70 & & $\cdots$ & 1991.25 & TYC2002 \\
\hline HD 83808 & & $\cdots$ & $1.6 \pm .2$ & & $\cdots$ & & 1997.07 & $\rho=0.6^{\prime \prime}$, ARI1999 \\
\hline HIP 83808 & $\ldots$ & & & $\ldots$ & & $\ldots$ & & \\
\hline \multicolumn{9}{|l|}{$09412+0954 \mathrm{Ac}$} \\
\hline HD 165341 & & $1.85 \pm .1$ & $1.75 \pm .1$ & & $1.44 \pm .1$ & & 1995.56 & $\rho=1.6^{\prime \prime}$ \\
\hline HIP 88601 & $2.37 \pm .15$ & & & $1.90 \pm .15$ & & $\ldots$ & 1991.25 & FAB2000a \\
\hline \multirow[t]{3}{*}{$18054+0232$} & $\ldots$ & & & 1.84 & & $\ldots$ & 1991.25 & HIP1997a \\
\hline & $\ldots$ & & & 1.8 & & $\ldots$ & 1991.25 & TYC2002 \\
\hline & $\ldots$ & & & 2.0 & & $\ldots$ & 1994.5 & WSI1999a \\
\hline HD 183912 & & $0.54 \pm .1$ & $1.18 \pm .1$ & & $1.86 \pm .1$ & & 1995.559 & $\rho=34^{\prime \prime}$ \\
\hline HIP 95947 & $\ldots$ & & & 2.07 & & $\ldots$ & 1991.25 & HIP1997a \\
\hline $19307+2758 \mathrm{AB}$ & $\ldots$ & & & 1.1 & & $\ldots$ & 1991.25 & TYC2002 \\
\hline
\end{tabular}


the precision of the parallax is bad (ESA 1997).

HD 183912 Aa: $\beta_{1} \mathrm{Cyg}$, is a new triple interferometric system: in addition to the known component at $0.4^{\prime \prime}$, Prieur et al. (2002) found another, close, companion at $0.05^{\prime \prime}$. GIN2002 gives K2II + B8:p. $d=118$ pc.

HD 186203: $\chi$ Aql, has a luminous cool primary: GIN2002 gives G2Ib + B5.5V. Bright Star Catalogue gives $\Delta m_{V}=1.2 . d=230 \mathrm{pc}$.

HD 186518, is a variable star (PS Vul). Bright Star Catalogue gives $\Delta m_{V}=1.5$. Very imprecise parallax (ESA 1997) and distance (1300 pc).

HD 187259: $\pi$ Aql, is a well known visual binary with a composite spectrum. Bright Star Catalogue gives $\Delta m_{V}=0.5 . d=175 \mathrm{pc}$.

HD 187321: McA 58, has a luminous cool primary: GIN2002 gives G5Ib-II + B7IV. $d \sim 1000$ pc (deduced from photometric model).

HD 194359: $d=265 \mathrm{pc}$.

HD 195692: has a primary of type Am that is a spectroscopic binary $(P=11.3$ days $)$. Bright Star Catalogue gives $\Delta m_{V}=1.8$.

HD 215242: is a variable star. Bright Star Catalogue gives $\Delta m_{V}=1.6$. GIN2002 gives the classification G8III + B7.5IV, but notes that the features of the IR spectra suggest, for the primary, a higher luminosity $\left(M_{V 1}=-0.6\right)$ than that of a normal giant. The parallax (ESA 1997) is very uncertain and so is its distance (1136 pc).

\subsection{Notes on the complementary list (Ta- ble 5)}

HD 74874 AC: we give here the photometry of the $\mathrm{AC}$ components. The $\mathrm{AB}$ components form the composite spectrum system listed in Table 4.

HD 83808 Ac: here also, AB pair is a composite spectrum star and a spectroscopic binary.

HD 165341: ADS 11046, is a binary star belonging to an astrometric program of orbit monitoring. It was observed with the CAR detector, with a different procedure (see Paper I) .

HD 183912 AB: Albireo, variable. Here, the composite spectrum couple is Aa (in Table 4). This system was also observed with the CAR detector.

\section{Discussion}

\subsection{Comparison with other measurements}

The confrontation of the data from PISCO observations with those obtained by other observers is illustrated in Fig. 8.

The magnitude differences obtained by PISCO and other sources given in Tables 4 and 5 are

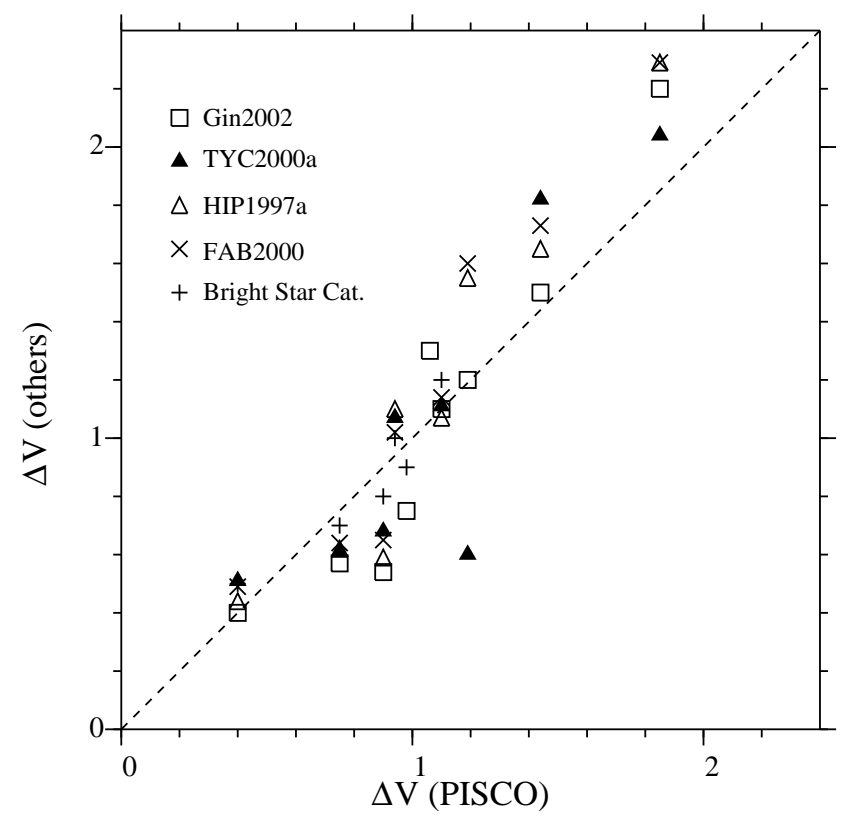

Fig. 8.- Comparison of PISCO differential photometric measurements with other measurements published in the litterature. All these data have been converted to Johnson system.

not in the same photometric system, which may explain some discrepancies in Table 4. Hence, in that figure, we have converted the measurements coming from Hipparcos satellite (HIP1997a, TYC2000a, and FAB2000) to the Johnson system using the formula given in ESA (1997) and the spectral types determined by Ginestet et al. (2002). Likewise, we have converted PISCO $\Delta V^{p}$ measurements to the Johnson system using corrections derived from the spectral models of Table 4 (Col. 2). No correction was applied to HD 12447, since the spectral types of both components are close (Am and $\mathrm{Ap}$ ).

The agreement is generally good for most objects, with a scatter of less than 0.2 mag.

\subsection{Comparison with spectral models}

As one can see in Table 4, the spectral types given by GIN2002 are in reasonable agreement with our measurements and those published in the litterature. For HD 8036, PISCO measurements would favour a cooler primary that GIN2002's model, but the difference is still large for $\Delta R^{P}$.

As our measurements are differential measurements, they are only sensitive to the difference $M_{V 2}-M_{V 1}$ of the absolute visual magnitudes of the primary $\left(M_{V 1}\right)$ and secondary $\left(M_{V 2}\right)$. The value of $M_{V 2}$ was taken from SchmidtKaler (1982), which we assumed to be well determined since the hot secondary was generally a 
dwarf star. Hence, our differential measurements allowed a direct determination of $M_{V 1}$, the visual magnitude to the cool component, generally a giant or a super-giant. The errors for $M_{V 1}$ are estimated at $\sim 0.6 \mathrm{mag}$ by taking into account the errors on the differential measurements (about 0.2 mag.), the errors on the determination of $M_{V 2}$ (about $0.5 \mathrm{mag}$ ) which is a combination of the noise in Schmidt-Kaler's calibration and the indetermination of the spectral type of the dwarf component.

In his paper, Pickles (1998) computed $M_{V}$ values from a fit of a population synthesis obtained with his spectral library to the 5 Gyr solar abundance isochrone by Bertelli et al. (1994). These values often led to unconsistencies with our measurements, especially for the giants with types around GK III. We therefore compared the values of the absolute magnitudes of the cool components found in Table 4 (Col. 11) with the calibrations proposed by various authors in Fig 9. Taking into account the value for the errors of $\Delta M_{V 1} \sim 0.6$ mag., we obtain a good agreement with Schmidt-Kaler (1982) and Keenan \& Barnbaum (1999), and a disagreement with Pickles' calibration of cool giants with population synthesis. Note that Keenan \& Barnbaum's new calibrations result from the contribution of the Hipparcos measurements: the absolute magnitudes of many cool bright giants around G6-K3 have been revised and decreased by about one magnitude relative to the values by SchmidtKaler. The G6-K3 cool giant stars whose visual absolute magnitudes remain close to 1.0, now form the group of IIIb giants (cf. Fig. 9).

\subsection{Comparison with Hipparcos total magnitudes}

In Table 6, we evaluate the consistency of our values of absolute magnitudes of individual components with the total absolute visual magnitudes measured by Hipparcos $M_{V}^{H}$. For each object of Table 4 for which a determination has been done, we give in Cols. 2 and 3 the values $M_{V 1}$ and $M_{V 2}$ of the primary and secondary components respectively (cf. Col. 11 of Table 4), and the corresponding total absolute magnitude $M_{V}$ of the system in Col. 4. We present in Col. 5 the Hipparcos measurements $M_{V}^{H, c o r r}$, which have been corrected for interstellar redening by GIN2002, and the corresponding error in Col. 6. The error $\Delta M_{V}$ that we can expect for $M_{V}$ from our measurements are estimated at $\sim 0.8 \mathrm{mag}$., since $\Delta M_{V 2} \sim 0.5 \mathrm{mag}$. and $\Delta M_{V 1} \sim 0.6 \mathrm{mag} .$. In all cases, except for HD 18925 and HD 186203, $\left|M_{V}^{H, c o r r}-M_{V}\right| \lesssim \Delta M_{V}^{H}$. For these two objects, the difference is 0.29 and 0.84 respectively, which

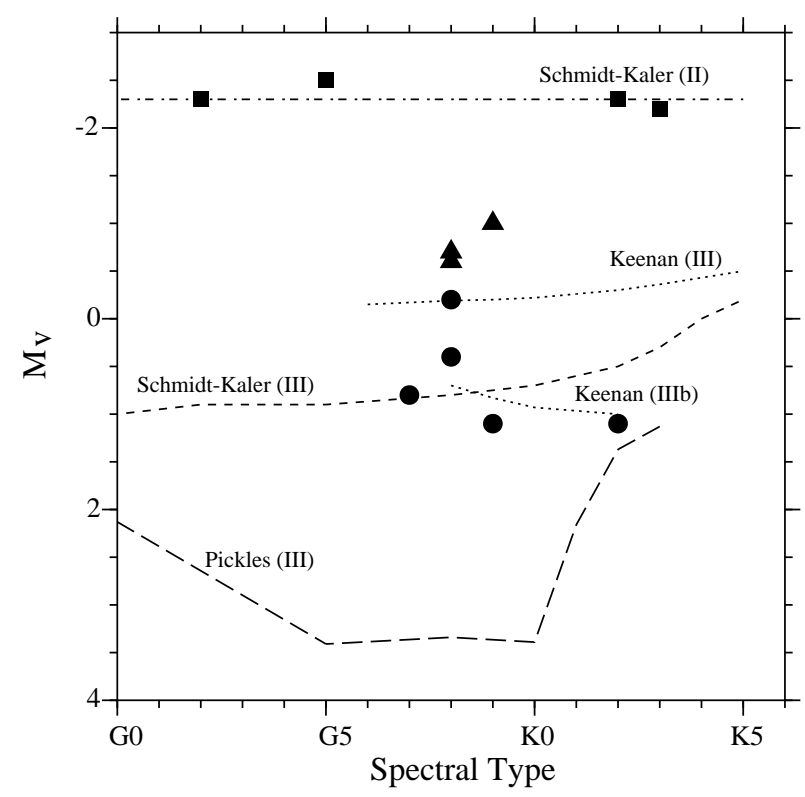

Fig. 9.- Absolute magnitudes of the cool components derived from our measurements for giants of class II (squares), II-III (triangles) and III (circles), compared to calibrations by SchmidtKaler (1982), Pickles (1998) and Keenan \& Barnbaum (1999).

is less than, or of the same order of, the errors on $M_{V}$. Hence, the total magnitudes $M_{V}$ derived from our study are compatible with Hipparcos data within the errors.

This comparison nevertheless shows that the spectral classification of HD 186203 as $G 2 I b-I I+$ $B 5 \mathrm{~V}$ is only marginally compatible with Hipparcos data, although it leads to a very good agreement with differential photometry data (cf. Table 4).

\section{Conclusion}

Over the last ten years, the PISCO speckle camera of Observatoire Midi-Pyrénées has proven to be well adapted to efficiently perform relative astrometry of binary and multiple stars. This work has shown it could also be used for photometric purposes.

The full process from the data acquisition of simulated astronomical targets to the restored images and final photometric measurements was successfully tested in the laboratory with an optical test bench experiment.

Confrontation of photometry obtained with PISCO on astronomical targets with data from other sources showed a very good agreement, with a typical difference of less than $0.2 \mathrm{mag}$ for individual components of close double systems. The absolute 
TABLe 6

Comparison with Hipparcos total absolute

MAGNITUDES

\begin{tabular}{lccccc}
\hline \hline $\begin{array}{c}\text { Name } \\
(1)\end{array}$ & $\begin{array}{c}M_{V 1} \\
(2)\end{array}$ & $\begin{array}{c}M_{V 2} \\
(3)\end{array}$ & $\begin{array}{c}M_{V} \\
(4)\end{array}$ & $\begin{array}{c}M_{V}^{H, \text { corr }} \\
(5)\end{array}$ & $\begin{array}{c}\Delta M_{V}^{H} \\
(6)\end{array}$ \\
\hline HD 8036 & 1.1 & 1.7 & 0.61 & 0.37 & 0.34 \\
HD 18925 & -1.0 & 0.1 & -1.34 & -1.63 & 0.12 \\
HD 74874 & 0.8 & 1.7 & 0.41 & 0.26 & 0.12 \\
HD 166479 & -0.7 & 0.2 & -1.09 & -2.58 & 1.73 \\
HD 183912 & -2.3 & -0.4 & -2.47 & -2.58 & 0.15 \\
HD 186203 & -2.3 & -1.2 & -2.64 & -1.80 & 0.43 \\
HD 186518 & -2.2 & -1. & -2.51 & -4.89 & 2.58 \\
HD 187259 & 0.4 & 0.8 & -0.17 & -0.59 & 0.42 \\
HD 187321 & -2.5 & 1.1 & -2.53 & $\ldots$ & $\ldots$ \\
HD 194359 & -0.2 & 1.0 & -0.51 & -0.47 & 0.59 \\
HD 215242 & -0.6 & -0.9 & -1.51 & -4.61 & 2.98 \\
\hline
\end{tabular}

visual magnitudes of the cool giant stars that we determined are in good agreement with SchmidtKaler (1982) and Keenan \& Barnbaum (1999).

We would like to thank the technical staff of the "Télescope Bernard Lyot" for providing assistance during the observations.

We are grateful to Denis Mourard who lent us the CAR detector.

\section{REFERENCES}

Aristidi E., Carbillet M., Lyon J.-F., Aime C., 1997a, A\&AS, 125, 13.

Aristidi E., Carbillet M., Prieur J.-L, Lopez B., Bresson Y., Koechlin L., 1997b, A\&AS, 126, $555-561$.

Aristidi E., Prieur J.-L., Scardia M., Koechlin L., Avila R., Lopez B., Rabbia Y., Carbillet M., Nisenson P., Gezari D., 1999, A\&AS, 134, 545552.

Batten A.H., Fletcher J.M., MacCarthy D.G., 1989, Publ. Dom. Astrophys. Obs. 17, 1

Bertelli G., Bressan A., Chiosi C., Fagotto F., Nasi E., 1994, A\&AS, 106, 275

Bidelman W.P., 1958, PASP 70, 168

Carquillat J.-M., Jaschek C., Jaschek M., Ginestet N., 1997, A\&AS, 123, 5.

Cowley A.P. 1976, PASP 88, 95
Dainty J.C., \& Greenaway A.H., 1979, JOSA, Vol. $69, \mathrm{~N}^{\circ} 5,786$.

Douglass G.G., Hindsley R.B., Worley C.E. 1997, ApJS 111, 289

Douglass G.G., Mason B.D., Rafferty T.J., Holdenried E.R., Germain M.E. 2000, AJ 119, 3071.

ESA 1997, "Hipparcos and Tycho Catalogues", ESA SP-1200.

Fabricius C., \& Makarov V.V., 2000, A\&A 356, 141.

Fabricius C., Hog E., Makarov V.V., Mason B.D., Wycoff G.L., Urban S.E. 2002, A\&A, 384, 180.

Germain M.E., Douglass G.G., Worley C.E., 1999a, AJ 117, 1905

Germain M.E., Douglass G.G., Worley C.E., 1999b, AJ 117, 2511

Germain M.E., Douglass G.G., 2001, AJ, 121, 2239.

Ginestet N., Carquillat J.-M., Jaschek M., Jaschek C., Pédoussaut A., Rochette J., 1992, Atlas de spectres stellaires, Obs. Midi-Pyrénées, Obs. de Strasbourg.

Ginestet N., Carquillat J.-M., Jaschek M., Jaschek C., 1994, A\&A, 108, 359.

Ginestet N., Carquillat J.-M., Jaschek C., Jaschek M., 1997, A \& A, Suppl., 123, 135. 
Ginestet N., Carquillat J.-M., Jaschek C., 1999, A \& A, Suppl., 134, 473.

Ginestet N., \& Carquillat J.-M., 2002, ApJS submitted.

Goodman J.W., 1985, "Statistical Optics", Willey Interscience.

Hartkopf W.L., Mason B.D., McAlister H.A., 1996, AJ 111, 370

Hartkopf W.L., Mason B.D., Wycoff G.L., McAlister H.A., 2002, "Fourth Catalog of Interferometric Measurements of Binary Stars" http://ad.usno.navy.mil/wds/int4.html

Hoffleit D., \& Jaschek C., 1982, Bright Star Catalogue, Yale Univ. Obs.

Hog, E. et al., Tycho-2 Catalogue, 2000, A\&A 357, 367

Horch E.P., Robinson S.E., Meyer R.D., van Altena W.F., Ninkov Z., Piterman A., 2002, AJ, in press.

Ismailov R.M., 1992, A\&AS 96, 375.

Keenan P.C., \& Barnbaum C., 1999, ApJ 518, 859.

Labeyrie A., Bonneau D., Stachnik R.V., Gezari D.Y., 1974, ApJ 194, L147.

Lannes, A., 1988, Proc. of the NOAO-ESO conference on High Resolution Imaging by interferometry II, (Ed. J.M. Beckers and F. Merckle) Garching, Germany, p 169.

Lannes, A., Roques, S., Cazanove, M.J., 1987a, J. Mod. Optics, 34, 161-226.

Lannes, A., Roques, S., Cazanove, M.J., 1987b, J. Mod. Optics, 34, 321-370.

Lohmann A.W., Weigelt G., Wirnitzer B., 1983, Appl. Opt., 22, 4028.

Nakajima T., 1988, JOSA A, Vol. 5, 1477.

Pickles A.J., 1998, PASP 110, 863.

Popper D.M., \& McAlister H.A., 1987, AJ 94, 700

Pourbaix D., 1999, A\&A 348, 127

Prieur J.-L., Lannes A., Cullum M., 1991, Proc. of the NOAO-ESO conference on High Resolution Imaging by interferometry II, (Ed. J.M. Beckers and F. Merckle) Garching, Germany, p 353.

Prieur J.-L, Koechlin L., André C., Gallou G., Lucuix C., 1998, Experimental Astronomy, vol 8, Issue $4, \mathrm{p} 297$.
Prieur J.-L., Koechlin L., Ginestet N., Carquillat J.-M., Aristidi E., Scardia M., Arnold L., Avila R., Festou M.C., Morel S., and Pérez J.P., 2002, ApJ Suppl., in press, Paper I.

Roberts L.C., 1999, PASP, 650, 1999

Scardia M., 1983, AN 304, 257

Schmidt-Kaler Th., 1982, in Landolt-Börnstein, New Series, Gr.6, Vol.2-B, 1 (Springer-Verlag Berlin).

Schröder K.-P., Pols O.R., Eggleton P.P., 1997, MNRAS, 285, 696.

Schoeller M., Balega I.I., Balega Y.Y., Hofmann K.-H., Reinheimer T., Weigelt G. 1998, SvAL 24, 283-287.

ten Brummelaar T.A., Mason B.D., McAlister H.A., Roberts L.C., Jr., Turner N.H., Hartkopf W.I., Bagnuolo W.G., Jr., 2000, AJ 119, 2403.

Tokovinin A.A., \& Gorynya N.A., 2001, A\&A 374, 227

Wirnitzer B., 1985, JOSA A, 2, 14.

Worley C.E., Mason B.D., Wycoff G.L., 2001, AJ, 122,3484 .

This 2-column preprint was prepared with the AAS LATEX macros v5.0. 